\title{
Will the "normality" times come back? L2 learning motivation between immigrants and refugees before Covid-19
}

\author{
[¿Volverán los tiempos de "normalidad"?: motivaciones para el \\ aprendizaje de la L2 de inmigrantes y refugiados previas al Covid-19]
}

\author{
Monica Ortiz-Cobo - Jose Garcia-Martin - Rosella Bianco
}

DOI: 10.18355/XL.2021.14.01.15

\begin{abstract}
In these turbulent times of changes and transformations where educational processes are being virtualized due to the pandemic, we must not forget the difficulties that this implies for certain vulnerable learners and some learning contexts. That is the case of the L2 learning by immigrants and refugees. Such learners already have a starting difficulty, both for not mastering the language of learning and for the digital divide, which is increased by the migration variable.

This work analyses, within a context of "normality", the motivation of immigrants and refugees, in the light of their links and expectations. The differences in the relation with the Italian language are shown. The results show that the dependency on the host society, the uncertainty of the future and the absence of family ties are influential factors for refugees learning motivation. On the contrary, immigrants are not subjected to these factors, and therefore their Italian L2 learning motivations are different. By this research we conclude that adult refugees and immigrant students have different attitudes through the residency country language learning.
\end{abstract}

Key words: Second language, motivation, refugees, forced migration, ethnography, pre-Covid-19

\section{Resumen}

En estos tiempos tan convulsos de cambios y transformaciones donde los procesos educativos se están virtualizando a causa de la pandemia no debemos olvidar la dificultad que ello supone para determinados aprendizajes y colectivos vulnerables. Es el caso del aprendizaje de la L2 del colectivo de inmigrantes y refugiados. Quienes ya cuentan con una dificultad de partida, por no dominar la lengua vehicular de aprendizaje y en los que la variable migración supone un factor que acrecienta la brecha digital. Este trabajo analiza en un contexto de "normalidad" la motivación de inmigrantes y refugiados, a la luz de sus vinculaciones y expectativas. Se evidencian las diferencias en las relaciones con la lengua italiana. En el caso de los refugiados, los resultados muestran que en dicha relación influyen la dependencia con la sociedad de acogida, la incertidumbre sobre el futuro y la ausencia de lazos familiares en el país de recepción. Por el contrario, los inmigrantes, no están sujetos a estos factores, diferenciándose en sus motivaciones para el aprendizaje del italiano. Hemos podido concluir que los estudiantes refugiados e inmigrantes adultos tienen diferentes actitudes hacia el aprendizaje de la lengua del país de residencia.

Palabras claves: Segunda lengua, motivación, refugiados, migración forzada, etnografía, pre-Covid-19

\section{Introducción}

Cuando se habla de lengua y de comunicación entre individuos, es importante indicar que la lengua no es solo un sistema de signos y elementos interdependientes, sino un acto y un producto social que requiere su investigación desde una mirada sociológica. Sociedad y lengua están conectadas por una relación de interdependencia. Es decir, la 
lengua es un producto de la sociedad, y a su vez, influye sobre las actividades sociales. Así que, el uso de la lengua está estrictamente condicionado por factores socioculturales.

En este sentido, la elección de hablar una lengua y no otra a partir del propio repertorio lingüístico viene dictada por razones relacionadas con el contexto sociocultural (Marcos Casquero, 1984). En el ámbito migratorio, esta elección está supeditada a menudo a decisiones políticas de carácter asimilacionista por las cuales los ciudadanos llamados 'extranjeros' tienen que ajustarse a la sociedad de acogida a través, entre otras cosas, del aprendizaje de su lengua (Locchi, 2012; Catarci, 2014).

A partir de estas consideraciones y teniendo en cuenta que el incremento de los flujos migratorios ha hecho poner el foco de atención en el aprendizaje de la lengua del país de recepción como medio para la integración, consideramos relevante plantear como objeto de estudio las relaciones que los ciudadanos inmersos en procesos de migración mantienen con las lenguas que usan y aprenden, especialmente con la lengua del país de acogida (Kobylarek, 2010). La relevancia radica en que la relación que los sujetos establezcan con las lenguas, y en particular con la lengua de acogida, influirá en la construcción de sus propias identidades y en la relación que establezcan con el contexto sociocultural, determinando su desarrollo personal.

En este sentido parece interesante investigar las relaciones establecidas con la lengua del país de acogida por parte de los migrantes forzados, cuyo objetivo migratorio (o destino deseado) no siempre corresponde con el país de la permanencia. Diferente es el caso de quien migra por razones económicas y familiares, los cuales tienen más poder de decisión sobre su recorrido migratorio. Las circunstancias que implican ambas situación de migración, la de los refugiados y los inmigrantes, determinan las motivaciones de aprendizaje de las lenguas relacionadas con el país de acogida, tal y como pretendemos mostrar con el estudio realizado y que pasamos a presentar.

\section{Migraciones y nuevas necesidades lingüísticas}

El colectivo de aprendices refugiados ha sido poco considerado en la literatura hasta ahora, probablemente porque el fenómeno del asilo y del refugio ha estado relegado al problema del control de la inmigración obviando las nuevas necesidades educativas (Pinson, Arnot, 2007).

En el contexto italiano, la didáctica del italiano como segunda lengua (L2) ha empezado recientemente a especializarse en el ámbito migratorio, mostrando interés por las nuevas necesidades educativas como la alfabetización de los inmigrantes no escolarizados (Borri, Minuz, Rocca, Sola 2014; Minuz, Borri, Rocca 2016; Minuz y Borri 2016) y la certificación de las competencias en la L2 de adultos inmigrantes (Rocca, 2008). Se están dando los primeros pasos hacia una toma de conciencia de la necesidad de especialización en el campo de la didáctica para refugiados (Aloisi y Perna, 2016). Ello se debe a la urgencia de formar docentes preparados para enfrentarse a las particulares necesidades de aprendizaje de este grupo de estudiantes (Bianco, Ortiz Cobo, 2019a). Por otro lado, está emergiendo la insuficiencia de los cursos de lenguas en la integración lingüística de los refugiados, debido al poco tiempo que suele dedicarse a la educación de este colectivo (Bianco, Ortiz Cobo, 2019b; Stranovska, Gadusova, 2020; Gadusova et. al, 2020)

Sin embargo, el reciente interés hacia la enseñanza en contextos migratorios se debe al aumento de los flujos migratorios y la adopción de políticas de integración que reclaman el conocimiento de la lengua nacional como requisito para obtener el permiso de residencia de larga duración (Sergio, 2011; Locchi, 2012). A través de estas políticas, Italia se ha alineado en la tendencia ya presente en otros países europeos, en los que la integración de los adultos inmigrantes pasa necesariamente por requisitos lingüísticos que regulan el acceso, la permanencia o la adquisición de la ciudadanía en un país dado. En el periodo comprendido entre 2009 y 2013, la

XLinguae, Volume 14 Issue 1, January 2021, ISSN 1337-8384, eISSN 2453-711X 
legislación a este propósito ha sido testigo a nivel europeo de un aumento de las leyes inherentes el aprendizaje lingüístico para fines migratorios (Pulinx, Van Avermaet, Extramiana, 2014).

Así pues en el contexto italiano, el Decreto Ley 4 Junio 2010 que establece los nuevos criterios para la permanencia del ciudadano extranjero en Italia prevé un conocimiento de nivel A2 de la lengua italiana (según el Marco Común Europeo de Referencia para las Lenguas), requisito imprescindible para quien solicita el permiso de residencia de larga duración y que gracias a este reducen las numerosas solicitudes para renovar permisos más cortos (Mastromarco, 2011). Según el Marco Común Europeo, el nivel A2 corresponde a un conocimiento básico de la lengua, que supone el uso de expresiones sencillas sobre asuntos de utilidad inmediata, cuales pedir y dar informaciones sobre uno mismo y su entorno (Corpas Arellano, 2018; Council of Europe, 2001).

$\mathrm{Al}$ requisito de conocimiento de la lengua están exentos los refugiados, para los cuales la renovación del permiso está garantizada por la perpetuación de las razones por las cuales se les ha concedido el permiso la primera vez. De hecho, ellos residen en el país de acogida bajo un régimen de protección, según los derechos garantizados por la convención de Ginebra de 1951. Todavía, el aprendizaje de la L2 queda considerado como uno de los pasos para lograr la integración, ya sea de los inmigrantes como de los refugiados, colocándose en el seno de una dinámica asimilacionista que, sin embargo, termina reservando a estos ciudadanos solamente roles marginales en la sociedad (Catarci, 2014).

En este contexto, brillan por su ausencia los estudios sobre la dimensión emocional implicada en la vinculación de los procesos de acogida e integración con la lengua, así como los estudios sobre la motivación de los refugiados en el aprendizaje del italiano L2.

Los usuarios extranjeros de los cursos de italiano han ido cambiando en las últimas décadas y las motivaciones de los usuarios inmigrantes no son las mismas que las de los estudiantes de italiano como lengua extranjera (Vedovelli, 2002; De Mauro, Vedovelli, Barni, Miraglia, 2003). Por lo que es necesario un análisis de los nuevos aprendices, conformados ampliamente por refugiados y solicitantes de asilo.

En esta dirección, es indispensable tener en cuenta algunos aspectos fundamentales. Es necesario diferenciar entre migraciones voluntarias y forzadas y las implicaciones que ellas tienen en el aprendizaje de la L2. Los refugiados constituyen un tipo particular de inmigración, su peculiar situación los coloca en un sistema de privaciones-obligaciones. Una vez llegados a Italia, son víctimas de retención administrativa (Campesi, 2011) y la lengua forma parte de una de las imposiciones a las que están a menudo sometidos. En este sentido, el rechazo a aprender la L2 podría ser una manera de manifestar su necesidad de autoafirmación (Galli, 2017). Por otro lado, destaca la riqueza del repertorio lingüístico de este colectivo, que sufre un proceso de ampliación a través de su larga trayectoria migratoria (Bianco, Ortiz Cobo, 2019a).

\section{La motivación para el aprendizaje lingüístico}

Otro aspecto que es imprescindible considerar en los procesos de aprendizaje de la L2 son las necesidades que influyen en la motivación de estos particulares colectivos de aprendices.

Es necesario señalar que la literatura sobre la motivación en el aprendizaje lingüístico está en su casi totalidad enfocada en el estudio relativo al aprendizaje de la lengua inglesa. Estudios recientes señalan que más del $70 \%$ de las investigaciones del sector se centran en el aprendizaje de esta lengua (Boo, Dornyei, Ryan, 2015).

Debido al estatus y a los particulares roles que el inglés recubre en el contexto global, es posible suponer que el aprendizaje de una lengua diferente del inglés (definida en literatura por el acrónimo LOTE, desde el inglés 'language other than English'), 
puede estar motivado por razones diferentes de las que mueven el aprendizaje del inglés. Por otro lado, si pensamos al ámbito escolar, el aprendizaje del inglés puede comportar en el estudiante un menor interés hacia el aprendizaje de otras lenguas. De hecho, debido a su estatus, es común pensar que no es necesario aprender otras lenguas ya que hablando inglés es posible comunicarse prácticamente con todo el mundo. Por otro lado, la particular posición de esta lengua, presente en los planes de estudios desde los primeros años escolares, hace que esta lengua sea parte integrante de la educación recibida durante la escolarización, por lo que en definitiva su aprendizaje no supone una elección derivada de motivaciones personales, como puede ocurrir con otras lenguas (Dornyei, Al-Hoorie, 2017; Dornyei, Csizer, Nemeth, 2006, Graddol, 2006).

Cabe señalar que la literatura en este campo de estudio se focaliza principalmente en el aprendizaje lingüístico de lenguas extranjeras, aprendidas en el ámbito escolar, en un país diferente del de la lengua de aprendizaje (llamada lengua extranjera o LE). Por el contrario, muy escasa ha sido la atención prestada al aprendizaje de una segunda lengua en el país de origen de esta lengua (llamada segunda lengua o L2).

Al abordar la motivación para el aprendizaje de una segunda lengua, hemos considerado las teorías fundamentales que vinculan el aprendizaje lingüístico con las necesidades y expectativas del aprendiz. Resulta necesario considerar el tipo de motivación que influye en el aprendizaje. En este sentido, el enfoque de Gardner (2001) y el modelo más novedoso en el campo, el de Dörnyei (2005) aportan consideraciones relevantes. El primero analiza dos tipos de orientaciones que influyen en la motivación: la orientación integrativa y la orientación instrumental. La integrativa concierne a la necesidad de acercarse a la población local. Mientras que la instrumental se refiere al uso de la lengua como instrumento para alcanzar un resultado práctico o finalidad funcional como la laboral (Gardner, 2001). Dörnyei amplia esta teoría añadiendo tres conceptos: Ideal L2 Self (referido a la identificación con la comunidad hablante la L2), Ought-to L2 Self (referido a las expectativas externas) y L2 Learning Experience (referido a la experiencia de aprendizaje) (Dornyei, 2005). Específicamente el Ideal L2 Self se refiere a la imagen del ideal hablante de la L2 que el estudiante querría ser en el futuro. El Ought-to L2 Self, en cambio, representa los atributos que un aprendiz debe tener para cumplir las expectativas de otras personas (por ejemplo, las expectativas de los padres). Por último, la experiencia de aprendizaje o L2 Learning Experience se refiere al impacto que la experiencia de aprendizaje tiene en la motivación; por ejemplo la relación con el docente, los compañeros de clase y los resultados escolares (You, Dornyei, 2014).

El peso que cada uno de estos factores tiene en la motivación es variable y cambia de un estudiante al otro. Por lo que se refiere al contexto migratorio, más en detalle en el aprendizaje de la segunda lengua por parte de refugiados, ha sido demostrado que el Ought-to L2 Self es un elemento de relieve en la motivación de este colectivo. Es decir, las presiones externas para aprender la lengua de acogida tienen, para este colectivo de estudiantes, una influencia relevante en el desarrollo de la motivación para el aprendizaje. (Polok et al., 2020) En detalle, estas presiones están ejercidas tanto por la comunidad de acogida que requiere que los refugiados comuniquen en la lengua local, como por el gobierno y las asociaciones de acogida que exigen la frecuencia y aprobación de cursos de la lengua nacional.

Por otro lado, es imprescindible en nuestro caso tener en cuenta las motivaciones que estimulan el aprendizaje de los adultos, tal y como el sueldo, la mejora de las condiciones de vida y el trabajo (Knowles, 2005). De igual modo adquieren una cierta importancia las teorías que relacionan la motivación con el tiempo de permanencia previsto en un país, porque cuando se planifica vivir en el país de la L2 por un largo plazo de tiempo se reduce la distancia social y ello puede favorecer la motivación para el aprendizaje de la lengua (Schumann, 1976).

XLinguae, Volume 14 Issue 1, January 2021, ISSN 1337-8384, eISSN 2453-711X 
Señalar que estos últimos aspectos, es decir, la experiencia de aprendizaje y el tiempo de permanencia previsto en un país pueden sufrir la influencia de la pandemia por Covid-19. De hecho, los presupuestos didácticos han sido completamente replanteados a causa de la pandemia, pasando de una didáctica presencial a las clases en línea. En el caso de algunos aprendices, este cambio representa una dificultad añadida para el aprendizaje ya que, como en el caso de los refugiados, el uso y la disponibilidad de medios tecnológicos son limitados debido a la peculiar condición de refugiado (Bianco, 2020). Por otro lado, el Covid-19 está teniendo un increíble impacto en las posibilidades de movilidad, comportando, por un lado, la imposibilidad de desplazarse hacia otro país debido a las restricciones impuestas para la prevención y contención del contagio y, por otro lado, la necesidad de volver al propio país de origen debido a las crecientes dificultades económicas. (Biana, Joaquin, Dacela, 2020). Por consiguiente, la motivación para aprender la lengua del país de permanencia puede aumentar o disminuir debido a los efectos de la Covid-19 en la permanencia en un país por parte de los sujetos migrantes. (Aguas, 2020; Biana, Joaquin, 2020a,b)

\section{Definición de los objetivos fundamentales del estudio}

Como ya hemos apuntado, nuestro interés por el objeto de estudio, está motivado, en primer lugar, por la escasa presencia de estudios, por un lado, sobre la motivación para aprendizaje de lenguas diferentes al inglés y por otro lado, a por las motivaciones para el aprendizaje de una segunda lengua, pues en este último caso los esfuerzos se han focalizado en el estudio del aprendizaje de lenguas extranjeras. No obstante, el incremento del fenómeno migratorio pone de manifiesto la necesidad de abordar este ámbito de estudio, por lo que desde este trabajo se analiza la motivación para el aprendizaje de una segunda lengua, diferente al inglés, concretamente la lengua italiana aprendida por sujetos inmigrantes y refugiados en Italia.

Así pues nuestra investigación se ha fundamentado en las teorías expuestas anteriormente, las cuales enmarcan este trabajo en el ámbito del aprendizaje lingüístico, y toma como fundamento los estudios sobre la motivación. El objeto teórico de estudio es el aprendizaje del italiano como segunda lengua en contextos migratorios y su relación con sus usos y motivaciones de los estudiantes.

El objetivo fundamental ha sido analizar, a través del método etnográfico, las relaciones que refugiados e inmigrantes adultos tienen con la lengua italiana y su aprendizaje. Para la consecución de este, se han planteado los siguientes objetivos específicos:

- Analizar la relación de los dos colectivos con el país de la L2, individuando diferencias entre el colectivo de los refugiados y el de los inmigrantes.

- Investigar la motivación para el aprendizaje del italiano e identificar las diferencias entre los dos colectivos.

- Analizar, a través de la comparación inmigrante-refugiado, las peculiaridades de los aprendices sujetos a migración forzada.

Para lograr estos objetivos, se ha analizado el complejo de relaciones de dichos aprendices con la lengua italiana y el país (Italia) de residencia. Además, se ha investigado directamente las motivaciones para el aprendizaje del italiano, teniendo en cuenta los proyectos de vida de los estudiantes en el país.

\section{Sobre el método de estudio}

Tradicionalmente para el estudio sobre la motivación para el aprendizaje de una lengua se ha utilizado técnicas cuantitativas basadas sobre la aplicación y análisis de cuestionarios. Sin embargo, en los últimos años, el creciente interés por este ámbito de estudio ha comportado la ampliación en los instrumento empleados, por lo que se ha utilizado también las técnicas cualitativas y mixtas (Boo, Dornyei y Ryan, 2015). 
Para realizar este estudio hemos adoptado un enfoque cualitativo, empleando una metodología etnográfica. Para la recogida de información se ha empleado el cuestionario, la observación participante y la entrevista informal. El uso del enfoque cualitativo ha permitido aproximarnos al contexto y a los sujetos de la investigación de manera natural, vivenciar una realidad para poder comprenderla e interpretarla. A través de un trabajo de campo prolongado nos hemos podido integrarnos en el contexto de investigación, conocerlo en profundidad, directamente y sin filtros (Alvarez Alvarez, 2008).

La elección de la metodología empleada ha estado determinada por la particularidad del contexto de estudio. En este sentido cabe señalar que aproximarse al colectivo de refugiados no ha sido fácil. De hecho, ellos asocian las situaciones en las que se les hacen preguntas con su relación con abogados y policía por las solicitudes de asilo. En este sentido, relacionarse de manera prolongada con ellos ha ayudado a establecer relaciones de confianza esenciales para el desarrollo de la investigación. Por otro lado, la presencia de analfabetismo y de bajos niveles de alfabetización así como de niveles educativos de los participantes ha supuesto una dificultad para la administración de los cuestionarios. (Kobylarek, 2019). El modo en que se ha solventado lo indicamos más adelante.

Con respecto a la observación, ella se ha realizado en 4 clases de italiano: 3 clases impartidas en un centro para la educación de los adultos y 1 clase impartida en un centro para la acogida de los solicitantes de asilo y refugiados. Las características de ambas entidades serán detalladas en el apartado siguiente, y se nombrarán a lo largo del artículo a través de códigos identificativos, con el objetivo de garantizar el anonimato y proteger la identidad de las mismas. Del mismo modo, se procederá con los sujetos informantes. Se utilizará el acrónimo CEA para referirnos al centro de educación de los adultos y CAR para el centro de acogida de los refugiados. Para nombrar a los informantes utilizaremos un código compuesto por una letra, ' $R$ ' para referirnos al colectivo de refugiados e 'I' para el colectivo de inmigrantes, seguida de un número para diferenciar informantes pertenecientes al mismo colectivo y, por último, se indicará el país de procedencia del informante (ejemplo: R2 Nigeria, I1 Brasil).

El periodo de observación participante se ha llevado a cabo durante dos meses en los cuales se ha asistido diariamente a las clases. La observación ha sido casi siempre dividida en tres o cuatro momentos: los que preceden y siguen a la lección, durante la lección y la pausa o descanso. Durante la lección, se ha entrado en contacto con el alumnado y con las dinámicas de clase y del curso, adoptando distintos roles: como observadora de la realidad presenciada, como participante interviniendo como docente que ha servido de apoyo a la responsable de la clase y de mediadora lingüística (actuando como traductora e intérprete). En otros momentos, ha sido posible entrar más en contacto con los docentes y conocer la rutina de la escuela. Durante las sesiones de observación participante se ha aprovechado para administrar el cuestionario, además de realizar entrevistas informales a los aprendices.

En cuanto al cuestionario, este consta de 54 preguntas de respuestas tanto abiertas como cerradas y ha sido estructurado en tres partes, la referida a la motivación y expectativas, la que aborda los usos de las lenguas habladas y la que pretende recoger la relación del estudiante con el aprendizaje del italiano. El cuestionario ha sido administrado de forma escrita y de forma oral en aquellos casos en los que el nivel educativo no permitiera la administración escrita. De hecho, algunos aprendices de la muestra están escasamente alfabetizados y otros, pese a estarlo, han tenido dificultades en la lectura y la comprensión de las preguntas. Por lo que algunos cuestionarios se han iniciado de manera escrita y continuados oralmente.

Con respecto a las entrevistas, han sido realizadas en varias sesiones, durante las mismas sesiones de observación participante. Se ha utilizado la entrevista informal,

XLinguae, Volume 14 Issue 1, January 2021, ISSN 1337-8384, eISSN 2453-711X 
que ha permitido de un modo natural y relajado lograr la visión subjetiva de los entrevistados, profundizando en su relación con la lengua italiana. Esto es importante porque nuestros informantes son particularmente sensibles a contextos más formales en los cuales se les administra una entrevista, ya que tienen experiencia con ella por motivos inherentes a la solicitud de asilo, lo que genera en ellos una gran aprensión. El uso de una modalidad más formal de entrevista condicionaría probablemente su actitud y respuestas. Por ello, se ha optado por un acercamiento de la manera más natural posible para no generar tensiones y lograr respuestas sinceras. Por el mismo motivo, ha sido necesario explicar bien el objetivo del cuestionario antes de su administración. Además hemos considerado utilizar la entrevista como medio para complementar el cuestionario y triangular los instrumentos de recogida de datos, pudiendo profundizar en los datos recogidos en el cuestionario, así como para confirmarlos.

En total, se han realizado 33 cuestionarios (10 inmigrantes y 23 refugiados) y 11 entrevistas (5 inmigrantes y 6 refugiados). Los cuestionarios se han repartido en 3 clases del CEA: 10 cuestionarios en la clase 1, 12 en la clase 2 y 11 en la clase 3 . Las 11 entrevistas se han realizado también en 3 clases: 3 en la clase 1, 3 en la clase 2 y 5 en la clase 3. Para ello, ha sido necesario el uso de diferentes lenguas, debido al bajo nivel de italiano de muchos estudiantes. Las lenguas utilizadas han sido el inglés, el francés, el portugués y el árabe. Estas lenguas han permitido la comunicación con la totalidad de los aprendices a excepción de dos estudiantes, pues uno de ellos únicamente hablaba criollo y el otro bengalí. En ocasiones nos hemos servidos de otros estudiantes en calidad de mediadores lingüísticos y hemos tenido que emplear más de una lengua. En concreto, los cuestionarios administrados de forma escrita se han pasado en inglés a 11 alumnos, en francés a 2 y en árabe a otros 2 . Por otro lado, 1 que se administró en francés se terminó realizando en italiano porque una vez leídas las cuestiones el estudiante se dio cuenta que tiene las suficientes competencias lingüísticas para realizarlo en la L2. Además otro administrado en portugués se realizó finalmente tanto en portugués como en italiano. Por otro lado, 4 han sido administrados en inglés, inicialmente realizados por escrito pero continuados oralmente. Los restantes han sido administrados oralmente: 2 en inglés, 8 en italiano, 1 en árabe e italiano y 1 en francés e inglés. En cuanto a las entrevistas, se han realizado 5 en italiano, 5 en inglés y 1 en francés.

\section{Sobre el contexto de estudio e informantes}

El trabajo de campo se ha desarrollado en dos entidades situadas en Salento (Italia): un centro que se ocupa de la acogida de los solicitantes de asilo y refugiados (CAR) y un centro que se ocupa de la educación de los adultos (CEA). Se han seleccionado por ser los centros a los que acuden este tipo de usuarios para aprender la lengua italiana. Además el CEA es la entidad principal donde se puede hacer el examen de lengua de nivel A2, requerido para la renovación del permiso de residencia de larga duración. Por su parte, el CAR se ocupa de la acogida de los solicitantes de asilo y de administrar cursos de lengua italiana, así como otras actividades además de facilitar alojamiento y comida. El CEA es una entidad pública encargada de la educación de los adultos a la que acuden adultos italianos o extranjeros para conseguir el certificado de educación primaria y donde también se administran cursos de lengua italiana para aprendices adultos de nacionalidad extranjera. En esta entidad, en los últimos años el perfil de los aprendices ha sido casi en su totalidad de estudiantes de nacionalidad extranjera: inmigrantes y refugiados. Los refugiados viven en centros de acogida de la zona y son enviados a estudiar italiano al CEA por los responsables del centro de acogida. Por eso, algunos estudiantes que asisten al curso del CEA son los mismos que asisten a los cursos organizados por el CAR. En este último, al ser un centro que solo atiende a refugiados, el perfil de los aprendices se reduce a este colectivo. 
Concretamente la recogida de información se ha realizado en los cursos de italiano que ambas entidades imparten, en 3 clases del CEA y en 1 del CAR. Como ya hemos adelantado anteriormente, 8 estudiantes del CAR, los cuales participan en este estudio como informantes, siguen el curso del CEA. Para estos casos se ha optado por recoger la información (a través de las entrevistas y cuestionarios) durante las clases del CEA, pues el tiempo del que se dispone en esta entidad es mayor. De hecho, ha sido posible realizar trabajo de campo al CEA 5 días a la semana porque se imparten clases a diario. Las clases de italiano del CAR tienen una frecuencia de 1 a 2 días por semana y se ha optado por dejar estos días solo para la observación. Todos los refugiados viven en centros de acogidas gestionados por entidades CAR. La selección de los sujetos ha estado dictada por cuestiones prácticas, se han entrevistado y administrado los cuestionarios a los sujetos que disponían de más tiempo en clase. De hecho, la mitad de los estudiantes estaban sujetos a los horarios de trenes, utilizando los transportes públicos para venir a clase, por lo que no lograban seguir la lección entera. Esto ha dificultado la recogida de la información, que se ha realizado en los momentos de pausas, así como en los momentos previos y posteriores a la lección.

En este contexto, cuando hablamos de refugiados incluiremos no solo los que ya poseen el estatus de refugiado, sino también a los solicitantes y no se hará distinción entre solicitantes o titulares de permiso de residencia por asilo político, por protección humanitaria o por protección subsidiaria. Nos referiremos a ellos, de manera general por ser todos individuos que por diferentes razones y riesgos graves, no pueden volver a su país y son víctimas de migración forzada. De la misma manera, cuando hablamos de inmigrantes no se diferenciará entre inmigrantes por razones económicas o familiares. Los que migran por motivos de estudio, en general, se dirigen a otros tipos de cursos organizados por las universidades $\mathrm{y}$, por tanto, quedan excluidos automáticamente.

Así pues, la muestra de este estudio está formada por 33 individuos de entre 16 y 40 años. De ellos 23 son refugiados y 10 son inmigrantes. Los dos colectivos están en Italia hace relativamente poco tiempo, por un periodo similar que oscila entre 1 mes y 3 años. La procedencia de los aprendices está repartida del siguiente modo: 2 aprendices de Somalia, 1 de Egipto, 1 de Marruecos, 1 de Mauritania, 1 de Guinea, 1 de la Costa de Marfil, 1 de Guinea Bissau, 3 de Gambia, 1 de Sierra Leona, 7 de Nigeria, 1 de Ghana, 1 de México, 1 de Colombia, 1 de Argentina, 1 de Brasil, 2 de Polonia, 1 de Ucrania, 2 de India y 2 de Pakistán. En suma, 18 aprendices proceden de África Occidental, 2 del Norte de África, 2 de África Oriental, 4 del Sur de Asia, 4 de América del Sur y 3 de Europa del Este.

\section{Análisis de datos \\ Motivaciones para el aprendizaje del italiano $\mathrm{L} 2$ de los refugiados}

$\mathrm{Al}$ abordar la motivación de los refugiados para aprender la lengua italiana, en primer lugar hemos tratado de determinar el motivo por el cual los informantes realizan un curso de italiano. La casi totalidad de los refugiados asisten a cursos de lengua simplemente porque el centro de acogida donde viven los derivan al curso que el mismo centro administra, así como a otros cursos externos. ()Es decir, en los programas de integración de los centros que atienden a refugiados en Italia la integración pasa por el aprendizaje de la lengua italiana, por lo tanto esta enseñanza ocupa un lugar relevante en los programas formativos de estos centros. La motivación para aprender italiano responde, por tanto, al cumplimiento de las expectativas del centro de acogida y forma parte, según el modelo de Dörnyei, de las motivaciones del grupo Ought-to L2 Self (Dornyei, 2005).

Para profundizar en el análisis de la motivación e intentar comprender la relación de los refugiados no solo con la lengua italiana sino también con el país de acogida, hemos considerado los proyectos de vida de estos aprendices, teniendo en cuenta las

XLinguae, Volume 14 Issue 1, January 2021, ISSN 1337-8384, eISSN 2453-711X 
expectativas del estudiante como futuro ciudadano o trabajador italiano. Atendiendo a estas variables hemos analizado las razones que les llevan a aprender la lengua italiana.

La casi totalidad de los refugiados entrevistados, 22 de 23 informantes, no tienen ningún lazo familiar en Italia y, a excepción de un caso, se encuentran solos en Italia, lo que supone una variable importante en el planteamiento de sus proyectos futuros. De hecho, los planes de vida de estos refugiados son bastante heterogéneos, denotando un sentimiento de incertidumbre por el futuro. Por ejemplo el informante R4 cuando hace referencia a dónde va a vivir en el futuro manifiesta: "Solamente el destino lo dirá, porque no depende de mí" (R4, Costa de Marfil).

Los proyectos de residencia de los refugiados son variados, sin embargo Italia es para muchos una tierra de paso, un refugio momentáneo. Aunque de entre los 23 refugiados hay quien piensa quedarse en Italia para siempre (concretamente 9 sujetos), el resto (14) se dividen entre quien no sabe todavía dónde vivir (8), quien se queda solo para terminar sus estudios (2), quien prevé volver un día a su propio país (2) y quien ya tiene planeado su destino en otro país (2).

Además, los proyectos de vida en otros países son considerados también por quien todavía no sabe qué hacer en su futuro y por quien ha decidido quedarse momentáneamente para estudiar. Es el caso, por ejemplo, del informante R5, que prevé vivir en Canadá o Alemania y que pretende vivir en Italia hasta que termine sus estudios:

Hasta cuando termine la Universidad. Porque quiero estudiar hasta la universidad. Esto me ayudará en mi futuro... Tomo este curso para mi futuro. Quiero estudiar en Italia y este curso me ayudará a continuar mi educación... Es muy importante escribir bien en italiano así que pueda sacar buenas notas en los exámenes (R5, Pakistán).

Además, también encontramos informantes que declaran querer volver a su país una vez que no haya más problemas en él y ven Italia como el refugio donde quedarse durante algunos años. Es el caso de 2 estudiantes, quienes a pesar de demostrar incertidumbre sobre su residencia futura, manifiestan la voluntad de volver a su propio país en un futuro, aunque este no sea próximo. Se trata de un estudiante egipcio y de un aprendiz de Ghana. En suma, solo 9 informantes planean quedarse de manera estable en Italia.

Al respecto es necesario apuntar que la elección de quedarse en Italia para siempre, en el caso de los refugiados, no está forzosamente ligada a una elección personal sino la única alternativa posible para huir de atrocidades y persecuciones, lo que implica que no se dé un aumento en la motivación por aprender la L2, como afirma la teoría de la distancia social de Schumann (Schumann, 1976). En consecuencia, no todos los que se quedan en Italia consideran este país como el país ideal donde vivir. Más bien son pocos los refugiados que mantienen motivaciones para aprender italiano por idealizar Italia y a los italianos y querer un día ser uno más o simplemente soñar con hacer carrera en Italia.

Así que 5 de los 6 entrevistados aprenden italiano por las expectativas de la sociedad y porque no tienen otra alternativa para comunicarse o encontrar un trabajo. Según el refugiado R6, no se puede vivir en Italia sin conocer la lengua, tal y como nos indica: "Si vives aquí sin la lengua no eres nada, de alguna forma" (R6, Ghana). De igual modo se manifiesta el aprendiz R7: "Es necesario aprender la lengua italiana porque los italianos no hablan inglés" (R7, Gambia).

Además, no siempre hay correspondencia entre el tiempo que se programa vivir en Italia y el tipo de motivación para el aprendizaje del italiano porque también entre los informantes que manifiestan quedarse en Italia de manera definitiva hay quien se siente obligado a aprender la lengua y se queda solo porque no tiene alternativas, no por una elección personal.

Haciendo referencia al modelo de Dörnyei, las motivaciones de los aprendices refugiados de nuestra investigación se pueden identificar con el tipo de motivación 
Ideal L2 self y Ought-to L2 self. Es decir, entre quien idealiza Italia y aspira a ser parte de esta comunidad de alguna manera y quien aprende italiano porque tiene que responder a expectativas sociales y se siente de algún modo obligado a hacerlo. Al respecto los estudiantes con una motivación de tipo Ideal L2 Self logran resultados más elevados en el aprendizaje, mientras que la motivación Ought-to L2 self, por el contrario, está ligada básicamente a sentimientos de obligación y no está necesariamente asociada a resultados positivos, como se recoge en estudios anteriores (Dornyei, Chan, 2013).

Es decir, mientras un grupo está realmente motivado por aprender la lengua porque hace de Italia su segunda casa y/o le gusta Italia, el otro grupo se siente obligado a aprender la L2 porque por el momento no tiene otras posibilidades y tiene que quedarse en Italia, aunque temporáneamente, por cuestiones burocráticas. Es lo que apunta el informante R8: "Es mejor Austria, allí hay trabajo. Yo fui en Austria unos meses, pero me hicieron volver, porque me han tomado las huellas dactilares aquí" (R8, Pakistán). No obstante, hay quien decidió quedarse en Italia porque este país le ha salvado la vida y por eso lo idealiza "Me siento muy bien en Italia porque los italianos son los que han salvado mi vida en el mar y me han traído aquí a Italia, me dan simplemente todo lo que necesito" (R9, Gambia). En estos casos, lo que parece influir es más la idealización de Italia como país que los ha acogido y salvado de atrocidades y de la privación de la libertad. Citamos algunos ejemplos al respecto: "Me siento bien porque nadie me niega mi libertad" (R4, Costa de Marfil); "Italia es mi futura casa después de que me hayan rechazado en mi país" (R10, Nigeria).

\section{Motivaciones para el aprendizaje del italiano L2 de los inmigrantes}

En el caso de los inmigrantes entrevistados, estos están casi todos en Italia con sus familias ( 9 sobre 10 informantes) y a veces están casados con italianos (es el caso de 4 informantes). Aunque ellos estén en Italia por motivos principalmente laborales, los lazos familiares parecen fortalecer sus vínculos con el país italiano.

Los proyectos de vida de los inmigrantes son más homogéneos que los de los refugiados: 9 de los 10 inmigrantes quieren quedarse en Italia, ya que su situación es estable y tienen un trabajo y/o una familia que los liga al territorio. Es el caso del informante I1 que lleva un año en Italia: "Mi casa ahora está aquí, mi marido es italiano y pronto vamos a tener hijos, sería muy complicado irse a otro país y además estamos muy bien aquí, la vida no cuesta mucho y la familia de mi marido me ha acogido como una hija" (I1, Brasil).

Dentro del colectivo de inmigrantes hemos podido diferenciar entre quien vive en Italia con parientes de su tierra de origen ( 5 de 10 sujetos) y quien se ha casado y vive con italianos (4 de 10). Solo en un caso, un inmigrante indiano, vive con amigos. Al contrario que los refugiados, todos los inmigrantes, con la excepción de un solo caso, quieren quedarse en Italia y por esta razón todos quieren aprender italiano. El único aprendiz que no está seguro sobre su permanencia futura en Italia es un ciudadano marroquí de 21 años, él aunque viva con parientes en Italia, piensa que Italia no le ofrece mucho en términos de trabajo y se plantea la posibilidad de irse a otro país, una vez haya aprendido inglés.

En suma, los factores que influyen en la motivación del colectivo de inmigrantes tienen que ver con el hecho de estar casado con una pareja italiana así como vivir con la propia familia de origen o con parientes. Es decir, la estabilidad familiar es para este colectivo el principal factor que determina su permanencia en Italia y la necesidad de aprender la lengua local. Además, las razones para aprender el italiano son instrumentales, ligadas a la carrera laboral y al reconocimiento de Italia como país ideal donde vivir. Las motivaciones de este colectivo por tanto son del tipo Ideal 2 Self, del modelo de Dörnyei (2010). Recogemos a este propósito las palabras del informante I1 (mujer casada con un hombre italiano): "Italia es un país que adoro, a

XLinguae, Volume 14 Issue 1, January 2021, ISSN 1337-8384, eISSN 2453-711X 
través de la lengua espero conocer más su cultura y sus tradiciones populares, es un país rico de cultura, adoro la música y quiero aprender a bailar la pizzica (baile local del sur Italia), es muy divertido" (I1, Brasil).

\section{Construcción de la motivación y los lazos con el país de acogida}

Durante la observación se ha evidenciado la influencia que la entidad de atención y/o acogida y sus docentes pueden tener en la motivación por aprender italiano, haciendo que pueda incrementarse o descienda. Aunque esto tenga validez para cualquier tipo de estudiante, creemos que en el caso de los refugiados es particularmente significativo, debido al estado psicológico del refugiado. De hecho, dicho estado está condicionado por factores como los traumas, haber vivido en duras condiciones o la preocupación por los familiares en que se encuentra en zona de guerra (Kirmayer et al., 2011). Además, experimenta una situación de transición y sufre largos periodo de espera para obtener el estatus de refugiado, que lo coloca una vez más en la incertidumbre.

Además, las relaciones que el refugiado tiene con la población local se limitan, en los primeros momentos, a los contactos que mantiene con los trabajadores y docentes de los centros de acogida. A través de ellos, el refugiado crea una relación con el nuevo país, influyendo en la voluntad de quedarse, así como en los proyectos y la idealización de su futuro. Por lo que la responsabilidad de las entidades de acogida no se limita a la función mediadora entre el refugiado y el país receptor, sino que en ella se encuentra connotaciones pedagógicas.

El caso de los inmigrantes es diferente, porque en la mayoría de las ocasiones ellos viven una situación ya definida, teniendo a menudo familia, trabajo y proyectos futuros. Al contrario, para los refugiados las clases de italiano es una de las actividades que llenan un vacío. En este sentido recogemos las palabras del informante refugiado R11: "Estoy cansado, porque no he hecho nada hoy. ¿Tú a mi edad hacías muchas cosas, verdad? Yo no puedo hacer nada”. (R11, Guinea Bissau). Por este motivo, las clases, así como la relación con el docente y los trabajadores de los centros de atención y/o acogida pueden crear no sólo la motivación para aprender la lengua, sino también ofrecer motivos para quedarse en Italia y establecer lazos.

\section{Conclusiones}

Al abordar en este trabajo las diferencias en la vinculación de los refugiados e inmigrantes con el aprendizaje del italiano L2, encontramos que una de ellas hace referencia al carácter del proceso migratorio, es decir, que la migración sea forzada o voluntaria, así como a la condición de migrante que se encuentra en el país de recepción solo o con familia. La segunda diferencia está ligada a la primera, en cuanto que los colectivos analizados demuestran que es mucho más probable que un refugiado no tenga lazos familiares en Italia, mientras que quien migra de manera voluntaria lo hace bien con la familia o porque tenga familiares (u otro tipo de redes) ya en el país de destino, en cuyo caso sea como sea la migración se debe a motivos laborales para mejorar su calidad de vida.

La peculiar situación de los refugiados que implica la dependencia a la organización de acogida, la falta de lazos personales, un sentimiento de incertidumbre e inestabilidad, se contrapone con la posibilidad de elegir y planificar un proyecto futuro por parte de los inmigrantes, sea por motivos económicos o familiares. Todos estos factores repercuten tanto en el ámbito lingüístico como en las vidas de los sujetos estudiados. En particular, hemos visto como estos aspectos tienen influencia sobre las motivaciones para aprender la L2.

Los resultados muestran que, debido a la gran incertidumbre en los proyectos de vida de los refugiados, muchos planean desplazarse a otros países o volverse a su propio país apenas las condiciones sean favorables y, por tanto, tienden a ampliar la distancia social con los nativos italianos, lo que implica una disminución de la motivación para 
el aprendizaje de la lengua italiana, tal y como apunta Schumann en su trabajo de 1976. La previsión de residencia de los refugiados en el territorio italiano es generalmente temporal y por este motivo están motivados, a veces, a aprender otras lenguas y estudian la lengua italiana porque sienten no tener otra opción para poder comunicarse con la población local y encontrar un trabajo.

Además, el aprendizaje del italiano resulta para este colectivo a veces más una imposición que una elección. La mayoría lo aprende porque los centros de acogida donde reside lo deriva a los cursos de L2, mientras que el colectivo de inmigrantes lo aprende por motivos instrumentales, motivaciones que según Dörnyei confluyen en las motivaciones del Ideal L2 Self, las que están más ligadas a resultados positivos de aprendizaje. Este colectivo, además, aprende la lengua italiana por motivos laborales así como familiares y lo hace con previsiones de establecerse de manera permanente en Italia. No obstante encontramos algunos casos de refugiados que están más motivados por aprender la lengua italiana, movidos por la idealización de Italia como país que les ha salvado la vida. Estos, junto al colectivo de inmigrantes, son los más motivados para el aprendizaje del italiano.

Sin embargo, la reorganización de la didáctica de las lenguas en el actual contexto pandémico a través de clases no presenciales comporta la necesidad de averiguar cómo este tipo de experiencia de aprendizaje ha influido en la motivación de los aprendices de origen migrante, por lo que se plantea la necesidad de seguir investigando en esta línea de interés.

Finalmente, después de haber constatado la incertidumbre presente en los proyectos de vida de los refugiados y la consiguiente escasa motivación en el aprendizaje de la L2, nos planteamos si en un mundo globalizado, donde los desplazamientos humanos seguirán caracterizando todas las sociedades, el futuro de un modelo de convivencia eficaz, armoniosa, justa, en definitiva intercultural, pasa, no por potenciar medidas de carácter asimilacionista que imponga el aprendizaje de la lengua del país de acogida, sino por una política lingüística que favorezca el desarrollo de competencias plurilingües en todos los ciudadanos, no solo en refugiados e inmigrantes, sino en la población nativa italiana, de manera que se hable al menos una lengua franca.

\section{Bibliographic references}

AGUAS, J. J. S. 2020. Critical thinking in this time of global pandemic. In: Philosophia, Philippines, vol. 21, n. 2, pp. 285-290. ISSN 2244-1875

ALOISI, E. - PERNA, A. 2016. Ataya, manuale di lingua italiana multilivello. Bergamo: Sestante.

ALVAREZ ALVAREZ, C. 2008. La etnografía como modelo de investigación en educación. In: Gazeta de Antropología, vol, 24, n. 1, pp. 1-15. ISSN 0214-7564

BIANA, H.T. - JOAQUIN, J. J. B. 2020a. COVID-19: The need to heed distress calls of healthcare workers, In: Journal of Public Health, United Kingdom, vol. 42, n. 4, pp. 853-854. ISSN 1741-3842

BIANA, H.T. - JOAQUIN, J. J. B. 2020b. The ethics of scare: COVID-19 and the Philippines' fear appeals. In: Public Health, vol. 183, pp. 2-3. ISSN 0033-3506

BIANCO, R. 2020. An analysis of refugees' use of ICT: languages and limitations. In: Sociología y tecnociencia, vol. 10, n. 2, pp. 95-105. ISSN 1989-8487

BIANCO, R. - ORTIZ COBO, M. 2019a. Adquisición formal e informal de las lenguas en procesos migratorios: comparativa entre el caso de inmigrantes y refugiados. In: Lengua y habla, 23. ISSN 2244-811X

BIANCO, R. - ORTIZ COBO, M. 2019b. The linguistic integration of refugees in Italy. In: Social Sciences, vol. 8, vol. 10, 284 p. ISSN 2076-0760

BIANCO, R. - ORTIZ COBO, M. 2019c. Refugees education: an ethnography of teaching experiences in Jordan. In: The European Proceedings of Social and Behavioural Sciences, vol. 60, pp. 31-38. ISSN 2357-1330

XLinguae, Volume 14 Issue 1, January 2021, ISSN 1337-8384, eISSN 2453-711X 
BOO, Z. - DORNYEI, Z. - RYAN. S. 2015. L2 motivation research 2005-2014: understanding a publication surge and a changing landscape. In: System, vol. 55, pp. 145-157. ISBN 0346-251X

BORRI, A. - MINUZ, F. - ROCCA, L. - SOLA, C. 2014. Italiano L2 in contesti migratori. Torino: Loesher. ISBN 9788857714738

CAMPESI, G. 2011. La detenzione amministrativa degli stranieri: storia, diritto, politica. In: Democrazia e diritto, vol. 3-4, pp. 177-225. ISSN 0416-9565

CATARCI, M. 2014. Considerazioni critiche sulla nozione di integrazione di migranti e rifugiati. In: REMHU, vol. 43, pp. 71-84. ISSN 1980-8585

CORPAS ARELLANO, M. D. 2018. Revisión de las escalas de niveles lingüísticos y comunicativos: modelos norteamericanos y europeos. In: Revista de lenguas modernas, vol. 29, pp. 11-35. ISSN 1659-1933

COUNCIL OF EUROPE 2001. Common European Framework of Reference for Languages: learning, teaching, assessment. Cambridge: Cambridge University Press.

DE MAURO, T. - VEDOVELLI, M. - BARNI, M. - MIRAGLIA, L. 2003. Italiano 2000. I pubblici e le motivazioni dell'italiano diffuso fra stranieri. Roma: Bulzoni. ISBN 8883197976

DORNYEI, Z. 2005. The psychology of the language learner: individual differences in second language acquisition. Mahwah, NJ: Lawrence Erlbaum. ISBN 0805860185

DORNYEI, Z. 2010. Researching motivation: from integrativeness to the Ideal L2 Self. In S. Hunston y D. Oake (Eds.), Introducing applied linguistics. Concepts and skills, London: Routledge. pp. 74-83. ISBN 0415447682

DORNYEI, Z. - AL-HOORIE, A. H. 2017. The motivational foundation of learning languages other than global English: theoretical issues and research directions. In: The modern language journal, vol, 101, n. 3, pp. 455-468. ISSN 15404781

DORNYEI, Z. - CHAN, L. 2013. Motivation and vision: an analysis of future L2 Self images, sensory styles, and imagery capacity across two target languages. In: Language learning, vol. 63, pp. 437-462. ISSN 1467-9922

DORNYEI, Z. - CSIZER, K, - NEMETH, N. 2006. Motivation, language attitudes and language globalisation: a Hungarian perspective. Clevedon, UK: Multilingual Matters. ISBN 978-1853598852

GADUSOVA, Z. - HASKOVA, A. - SZARSOI, D. 2020. Teachers`competences evaluation: Case study. In: Science for Education Today, vol. 10, 3, pp. 164-177. ISSN 2658-6762.

GALLI, T. 2017. La logica del sistema di accoglienza dei richiedenti protezione internazionale e la sua ricaduta sull'apprendimento dell'italiano. In: Bollettino Itals, vol. 68, pp. 14-38. ISSN 2280-6792

GARDNER, R. C. 2001. Integrative motivation: past, present and future. Distinguished lecturer series. In Temple University Japan. Tokyo, Osaka. Recuperado, el 24 de abril de 2019, Available online: http://publish.uwo.ca/ gardner/docs/GardnerPublicLecture1.pdf

GRADDOL, D. 2006. English next: why global English may mean the end of 'English as a foreign language'. London: British Council.

IBRAGIMOVA, T. - SARGAZIN, Z. - ZHANZHUMENOVA, A. - AKCHIN, A. FEDOSEENKO, K. 2020. The issues of influence of moral denotions to self-esteem of people. In: Slavonic pedagogical studies journal. vol. 9. N. 1. pp. 86-92. ISSN 1339-8660 doi: 10.18355/PG.2020.9.1.10

JOAQUIN, J. B. - BIANA, H.T. - DACELA, M. A. 2020. The Philippine Higher Education Sector in the Time of COVID-19. In: Frontiers in Education, vol. 5, 576371. ISSN 2504-284X

KHUKHLAEV O.E. - GRITSENKO V.V. - PAVLOVA O.S. - TKACHENKO N.V. - USUBIAN S.A. - SHOROKHOVA V.A. 2020. Comprehensive Model of Intercultural Competence: Theoretical Substantiation. RUDN Journal of Psychology 
and Pedagogics, vol. 17, N. 1, pp.13-28. ISSN 2313-1705. doi: 10.22363/2313-16832020-17-1-13-28.

KIRMAYER, L. J. - NARASIAH, L. - MUNOZ, M. - MEB, R. - RYDER, A. G. GUZDER, J. - POTTIE, K. 2011. Common mental health problems in immigrants and refugees: general approach in primary care. In: Canadian medical association journal, vol. 183, pp. 959-967. DOI: https://doi.org/10.1503/cmaj.090292

KOBYLAREK,A. 2010. Integration of Elderly Citizens through Learning. In: New E ducational Review, vol. 22, n. 3-4, p, 24. ISSN 1732-6729

KOBYLAREK, A. 2019. Education in the post-scientific Culture. Journal of Education culture and Society, vol. 10, 1, pp. 5-13. ISSN 2081-1640

KNOWLES, M. S. - HOLTON, E. F. - SWANSON, R. A. 2005. The adult learner. San Diego: Elsevier. ISBN-13: 978-1856178112

LOCCHI, M. C. 2012. L'accordo di integrazione tra lo stato e lo straniero. (Art. 4-Bis T.U. sull'Immigrazione N. 286/98 alla luce dell'analisi comparata e della critica al modello europeo di 'integrazione forzata'. In: Associazione italiana dei costituzionalisti, vol. 1.

MALA, E. - MUGLOVA, D. - STRANOVSKA, E. 2019. Stimulation of creativity and ambiguity tolerance as effective foreign language teaching predictors. In: Slavonic pedagogical studies journal. vol. 8. n. 1. pp. 164-175. ISSN 1339-8660.

MARCOS CASQUERO, M. del C. 1984. Acercamiento a la sociolingüística. Aldaba, 2. ISSN 0213-7925

MASTROMARCO, A. 2011. Il test di lingua italiana 'scritta' per gli stranieri. In: Bollettino Itals, vol. 38 .

MINISTERO DELL'INTERNO. 2010. Decreto 4 giugno 2010. Modalità di svolgimento del test di conoscenza della lingua italiana, previsto dall'articolo 9 del decreto legislativo 25 luglio 1998, n. 286, introdotto dall'articolo 1, comma 22, lettera i) della legge n. 94/2009. Gazzetta Ufficiale, 134. Recuperado, el 24 de abril de 2019, Available online: http://3.flcgil.stgy.it/files/pdf/20100604/decreto-interministerialedel-4-giugno-2010-modalita-di-svolgimento-test-lingua-italiana-4819391.pdf

MINUZ, F. - BORRI, A. 2016. Literacy and language teaching: tools, implementation and impact. In: Italiano LinguaDue, vol. 2, pp. 220-231. DOI: https://doi.org/10.13130/2037-3597/8184

MINUZ, F. - BORRI, A. - ROCCA, L. 2016. Progettare percorsi di L2 per adulti stranieri. Torino: Loesher. ISBN 9788820137571

POLOK, K. - MALA, E. - MUGLOVA, D. 2020. On the salience of becoming a creative foreign language teacher. In: XLinguae, vol. 13, n. 4, pp. 152-162. ISSN 1337-8384.

PINSON, H. - ARNOT, M. 2007. Sociology of education and the wasteland of refugee education research. In: British Journal of Sociology of Education, vol. 28, pp. 399-407. ISSN 0142-5692

PULINX, R. - VAN AVERMAET P. - EXTRAMIANA, C. 2014. Linguistic integration of adult migrants: Policy and practice. Final report on the 3rd Council of Europe Survey. Strasbourg: Council of Europe. Recuperado, el 24 de abril de 2019, Available online: https://languageforwork.ecml.at/Portals/48/ICT_REV_LFW/LIAMSurveyReport2014_EN.pdf.pdf

RESKIANA - HARYANTO - WEDA, S. 2020. A study on vocabulary teaching strategy for students with impairment at special education school (SLB), Makassar, Indonesia. In: Slavonic Pedagogical Studies Journal, vol. 9, n. 2, pp. 141-151

ISSN 1339-8660.

ROCCA, L. 2008. Percorsi per la certificazione linguistica in contesti di immigrazione. Perugia: Guerra Edizioni. EAN 9788855701860

XLinguae, Volume 14 Issue 1, January 2021, ISSN 1337-8384, eISSN 2453-711X 
RUBACHA, K. - SIROTOVA, M. - CH MCZYNSKA-RUBACHA, M. 2016. Educational self-efficacy in teachers of various ethical orientations. In: New Educational Review. vol. 43, N. 1, pp. 193-200. ISSN 1732-6729.

SERGIO, G. 2011. Un lasciapassare per l'Italia. La legge Maroni e l'obbligo del test di italiano per stranieri. In: Italiano LinguaDue, vol. 1, pp. 53-64. DOI: https://doi.org/10.13130/2037-3597/1228

SCHUMANN, J. H. 1976. Social distance as a factor in second language acquisition. In: Language learning, vol. 26, pp. 135-143. ISSN 1467-9922

STRANOVSKA, E. - GADUSOVA, Z. 2020. Learners' success and self-esteem in foreign language reading comprehension. In: Education and Self Development, vol. 15, n. 3, pp. 109-119. ISSN 1991-7740.

VEDOVELLI, M. 2002. L'italiano degli stranieri: storia, attualità e prospettive. Roma: Carocci. ISBN 9788843023103

YOU, C. - DORNYEI, Z. 2014. Language learning motivation in China: results of a large-scale stratified survey. In: Applied Linguistics, vol. 37, pp. 495-519. ISSN 1477450X

Words: 8948

Characters: 56765 (31,54 standard pages)

Mónica Ortiz Cobo

Faculty of Political Sciences and Sociology

Department of Sociology

University of Granada

C/Rector López Argüeta, S/N

18007 Granada,

Spain

monicaoc@ugr.es

Prof. Dr. José García Martín

Faculty of Political Sciences and Sociology

Department of Sociology

University of Granada

C/Rector López Argüeta, S/N

18007 Granada,

Spain

jgarciamartin@ugr.es

Rosella Bianco* (corresponding author)

Institute of Migrations

University of Granada

Polígono Tecnológico Ogíjares

Calle Zamora. Parcela 111-112

18071 Granada,

Spain

rosellabianco@correo.ugr.es 